\title{
New opportunities of computer assessment of knowledge based on fractal modeling
}

\section{Svetlana Dvoryatkina $^{a}$, Eugeny Smirnov ${ }^{b}$, Arseny Lopukhin ${ }^{c}$}

${ }^{a}$ Institute of Mathematics, Natural Sciences and Technology, Yelets State University named after I. A. Bunin, Russia, ${ }^{b}$ Physical and mathematical department, Yaroslavl State Pedagogical University named after K. D. Ushinsky, Russia, ' International law department, Moscow State Institute of International Relations (university) of the Ministry of Foreign Affairs of the Russian Federation, Russia

\begin{abstract}
In this article the urgent problem of control systems modeling of professional competences and mathematical knowledge assessment of students is discussed. Pedagogical expediency in management of students cognitive activity by using of new informational technologies instruments in monitoring and assessment of knowledge is proved. The possibility of fractal methods application in perfecting of the system of computer monitoring of mathematical knowledge of students as a part of the adaptive training systems is considered and realized. The design of the technology of computer monitoring of students knowledge on the basis of fractal methods includes the following stages: development of cross-disciplinary fractal and organized base of key mathematical concepts; the creation of the expansible bank of educational and cognitive and research tasks, which is coordinated with fractal structure of the conceptual framework; the development of the program module, which is focused on individual estimation of quality of students' educational cognitive activity in two characteristics - depth of knowledge based on the Hurst exponent and the size of synergetic effect of educational cognitive activity. The software realization of technology of the computerized control of training in mathematical disciplines quality as a part of the adaptive training system is enabled in the programming language C\#. The experience of implementation and operation of the controlling systems realized in the adaptive training systems based on the fractal model operation showed reliability of their work and allowed to increase the quality of educational process management and its effectiveness in general. Using of fractal techniques in computer assessment of mathematical knowledge of students makes it possible to increase the accuracy and speed of evaluation of the students knowledge, to diagnose a wide range of cross-disciplinary knowledge within one discipline.
\end{abstract}

Keywords: Qualimetry, fractal, assessment of knowledge, training of mathematics.

Thanks: work was carried out with the support of RNF, the project № 16-18-10304. 


\section{Introduction}

Monitoring of professional competences formedness and evaluation of trainees` knowledge is one of productive methods of the modern student teaching and the necessary mechanism of monitoring of didactic system functioning and its development. The leading role in monitoring and diagnostics is assigned to the teacher. The teacher solves a complex problem of obtaining information about students as about the subject of tutoring, a condition of their experience, their personal qualities, knowledge, abilities, skills. It is necessary for the purpose of ensuring effective management of cognitive activity and development of the personality of the student. The modern level of development of the informational and communication technologies (CT) allows readdressing this function to computer systems, i.e. there is an evolutionary transition "traditional monitoring monitoring with use of technical means - computer monitoring".

Computer monitoring and formation of professional competences evaluation is the program module in the automated training system (ATS). "ATS is a computer ergatic system intended for optimization of the tutoring process with the use of informational and communication technologies instruments, based on the automation of the processes of the trainee`s activity management" [Robert (2012)]. Close integration of tutoring process and monitoring is implemented in the modern automated training systems, which are constructed with the application of artificial intelligence methods. The method of stage-bystage specification and activization of trainees` knowledge is the basis for the technology of such models creation. The possibility of individualization of tutoring is one of the most important advantages of use of ICT in the automated systems [Robert, 2010; Uglev, 2010]. By dint of adaptive and intellectual technologies the training system considers individual abilities of the student, his previous and current knowledge, abilities.

Questions of pedagogical monitoring and assessment of knowledge are developed by the famous scientists - A. I. Berg, V. A. Bolotov, B. Y. Likhttsinder, I. Y. Lerner, etc. Significant results were received in the field of structuring test tasks, creation of adaptive testing technologies (V. S. Avanesov, A. M. Mayorov, J. Kettel, E. Tornodayk, A. G. Shmelyov, J. Fischer, etc.) [Avanesov (2005), Shmelyov (2013)]. Today are developed and widely adopted new methods of diagnosis of students' knowledge and control of the training results at an ICT basis, in particular, automated systems for the control of experts training quality, electronic systems of a qualimetry of professional knowledge, the automated systems of test monitoring (SAT system), rating systems of assessment of knowledge, etc. (I. I. Kibzun, V. L. Latyshev, V. V. Myakishev, I. D. Rudinsky, U. M. Neumann, I. V. Robert, etc.) [Kibzun (2006), Latyshev (2009), Myakishev (2002), Rudinskiy (2005), Robert (2012) ]. These techniques allow estimating the level of assimilation of a training material not only quantitatively, but also qualitatively. The 
methods of mathematical statistics, a decision theory, a fuzzy logic and artificial intelligence are a methodological basis of such systems .

The interactive system of a qualimetry of students knowledge on the basis of fractal methods allows increasing to a significant degree the accuracy, the speed and the depth of evaluation of the students` training level, as well as the intelligent systems and integrative qualities operations, which allow developing and applying interdisciplinary knowledge and skills in their professional activity. The essence of the use of fractal techniques in the diagnosis is the symbiosis of qualitative and quantitative assessments of mastering complex structures of educational elements. This approach is especially important for a qualimetry of mathematical education where naturally arising multistage abstractions of object contents create conditions for development of the complex mathematical structures.

Another important aspect, that was not considered in the diagnosed techniques earlier, is the question of assessment of synergetic effect of the experience increase and personal development of the trainee resulting from educational cognitive activity. This effect is due to the coherent and coordinated in space and time action of the different in their nature operational mechanisms, which lead to high-quality changes in the student's personality. This synergistic effect is shown as an additional result in a context of integration of scientific knowledge in general and expansion, deepening of the internal structure of selforganization of mathematical knowledge in particular.

Integration of mathematical, humanitarian and natural science knowledge during development of the difficult educational elements is inseparably linked with a problem of the organization of monitoring. The key parameters are the speed and accuracy of the efficiency evaluation of production procedures and current processes. Improvement of these parameters is possible due to the use of ICT achievements. However, the estimated parameters are qualitative character. The quantitative data are necessary for computer processing. The developed methods allow to digitize the obtained results, including the amount of the synergetic effect. Thus, the author's proposed solution is very relevant and productive in the definition and qualification of "zones of proximal development." 


\section{Methods}

Let's consider the possibility of application of fractal methods in perfecting of system of computer monitoring of trainees` mathematical knowledge as a part of ATS. The first stage of the control system modification is the development of structure of system of key mathematical concepts with application of fractal methods, based on basic properties of fractals - self-similarity and preservation of an invariant. Moreover, by means of fractal structuring of the training mathematical material the process of establishment of forced correlation of the information space clusters at the different levels of fractal structures interpenetration takes place. This process allows establishing the level and depth of crossdisciplinary communications between the studied descriptors.

So, we consider educational and conceptual constructs as developing self-similar structures, that reflect the self-similarity of the integral in all its divisible parts in connection with an identical circuit of construction of all structural elements of the education content. The choice of a fractal set is proved by the fact that the fractal is a suitable construct of efficient compression and packing of the acquired information in its structure. Identifying mathematical concepts with a fractal set, for example, with The Sierpinski triangle, we have an opportunity to translate idea of the degree of connection of concepts into the language of geometrical images and an opportunity to visually illustrate a coupling of all concepts. The model of a building block of knowledge (mathematical concept) has an appearance:

$$
S=\Delta \cap\left(\Delta_{1}^{1}+\Delta_{1}^{2}+\Delta_{1}^{3}\right) \cap\left\{\left(\Delta_{2}^{1}+\Delta_{2}^{2}+\Delta_{2}^{3}\right) \cup\left(\Delta_{2}^{4}+\Delta_{2}^{5}+\Delta_{2}^{6}\right) \cup\left(\Delta_{2}^{7}+\Delta_{2}^{8}+\Delta_{2}^{9}\right)\right\},
$$

where $\Delta-$ the basic mathematical concept corresponding to the level of fundamental knowledge; $\Delta_{1}^{1}, \Delta_{1}^{2}, \Delta_{1}^{3}$ - the concepts formed after the first iteration (level of allvocational training), and corresponding to interobject, humanitarian and naturally scientific fields of knowledge respectively; $\Delta_{2}^{1}, \Delta_{2}^{2}, \Delta_{2}^{3}-$ the concepts formed after the second iteration (level of practical self-realization) and corresponding to intersubject domain; $\Delta_{2}^{4}$, $\Delta_{2}^{5}, \Delta_{2}^{6}$ - the concepts formed after the second iteration (level of practical selfrealization) and corresponding to humanitarian field; $\Delta_{2}^{7}, \Delta_{2}^{8}, \Delta_{2}^{9}$ - the concepts formed after the second iteration (level of practical self-realization) and corresponding to the scientific field of knowledge. At the same time the training material presentation is made so that there is a change, building and improvement of the available mathematical structures, transition to higher step of the organization, when the structure created earlier becomes substructure new and wider. We consider the process of formation of mathematical concepts structure, taking into account the allowed time of tutoring, to the third level with the degree of structure complexity approximately equal 1,6. It is possible to 
construct structure of the tutoring content, having brought process of iteration to several orders. However, such division does structure too unwieldy, confusing the main maintenance of educational area.

We apply the coefficient of depth of assimilation of a concept and its interrelation with other concepts for the quantitative assessment of the quality of assimilation of a training material (or an index of a cross-disciplinary connectedness of concepts). This index is expressed through the fractal dimension of the D stream of informational cluster (the density of filling of clusters). Fractal dimension is automatically calculated by ATS through The Hurst exponent. Fractal dimension of D is related to The Hurst exponent a prime ratio of $\mathrm{D}+\mathrm{H}=2$.

The calculation of the Hurst exponent establishing a persistence measure (tendency of process to trends) allows predicting of the dynamics of assimilation of a training material.

The Hurst exponent can be connected with the coefficient of normalized range $R / S$ where $\mathrm{R}$ - a range of the corresponding time series, $\mathrm{S}$ - a standard deviation. Let's consider an algorithm of calculation of The Hurst exponent for a one-dimensional time series $\xi(n)$ :

There is a one-dimensional time series $\xi(n)$ where $n$ - number of some casual event corresponds to a discrete instant of $t(n)$.

1. Let $\xi(n)$ be a quantity of cross-disciplinary concepts, that are connected with assimilation of one key concept, on $\boldsymbol{n}$ step on selection by length $\boldsymbol{k}$, where $\boldsymbol{k}$ is the period of sampling of all time frame of $\boldsymbol{T}$, given for studying, including classroom and unsupervised activities. Frequency of sampling is equal: $k=\frac{T}{n}, \quad \boldsymbol{n}$ is the number of measurements.

The number of the acquired concepts is defined by quantity of truly solved tasks.

2. We define the mean value of a measuring on $\boldsymbol{n}$ step during sampling of $\boldsymbol{k}$ :

$$
\bar{\xi}(n, k)=\frac{\sum_{j=1}^{n} \xi_{j}}{k} ;
$$

3. We define a deviation of a number of measurements $\xi(n)$ from the mean value:

$$
X_{j}(n ; k)=\xi_{j}(n)-\bar{\xi}(n ; k)
$$

4. We find a range:

$$
R(n ; k)=\max _{r \leq n} \sum_{j=1}^{r} X_{j}(n ; k)-\min _{r \leq n} \sum_{j=1}^{r} X_{j}(n ; k)
$$


5. We define a standard deviation of the considered time series:

$$
S(n ; k)=\sqrt{\frac{\sum_{j=1}^{n}\left(\xi_{j}-\bar{\xi}(n ; k)\right)^{2}}{k}} ;
$$

6. The Hurst exponent is defined from the formula

$$
\frac{R}{S}=\left(\frac{n}{2}\right)^{H} ;
$$

7. Communication of The Hurst exponent and dimension D of Hausdorff-Bezikovich is expressed by equality $\mathrm{D}=2-\mathrm{H}$.

The following stage is the development of an assessment technique of the synergetic effect of knowledge and activity integration, based on the process of educational elements assimilation. The most universal mathematical model of assessment of the synergetic effect of knowledge and activity integration is the model, in which cumulative effect of any activity is expressed as the sum:

$$
E=E_{s}+\sum_{i} E_{i}
$$

where $E$ is a cumulative effect of educational cognitive activity, $E_{s}$ is a synergetic effect, $E_{i}$ is an effect of educational cognitive activity at assimilation $i$, that is an element of mathematical knowledge.

A synergistic effect can be expressed in the following aspects:

- at the same amount of time, which is allowed for studying the structural elements of mathematical knowledge, there has been increase in the knowledge volume and achieved the possibility of "hidden knowledge", objectively existing, but subjectively unknown to the trainee. This cumulative effect is formed due to completeness of formation of abilities to operate with concepts in the solution of applied and professional tasks by means of establishment of cross-disciplinary communications at more deep levels of integration;

- at the same intellectual, physiological, informational resources dialogue of mathematical, scientific, humanitarian and informational cultures provides high-quality enrichment of intellectual operations, streamlining of contents and structure of cognitive experience, efficient development of research activity, self-control of personal lines of the student during learning the composite knowledge structures [Dvoryatkina (2016) ].

Geometrical representation of a distribution in frequency of conceptual space is the cornerstone of the technique of estimation of size of synergetic effect. The closed set on the 
plane limited by the circle, which is circumscribed around an equilateral triangle, represents the figure, that allows estimating volume of knowledge. This volume of knowledge is in the vicinity of the central core and includes both the obviously mastered material (obligatory, provided) and the conditionally mastered material (not obligatory, variable), that gets into the field of development in a random way (fig. 1).

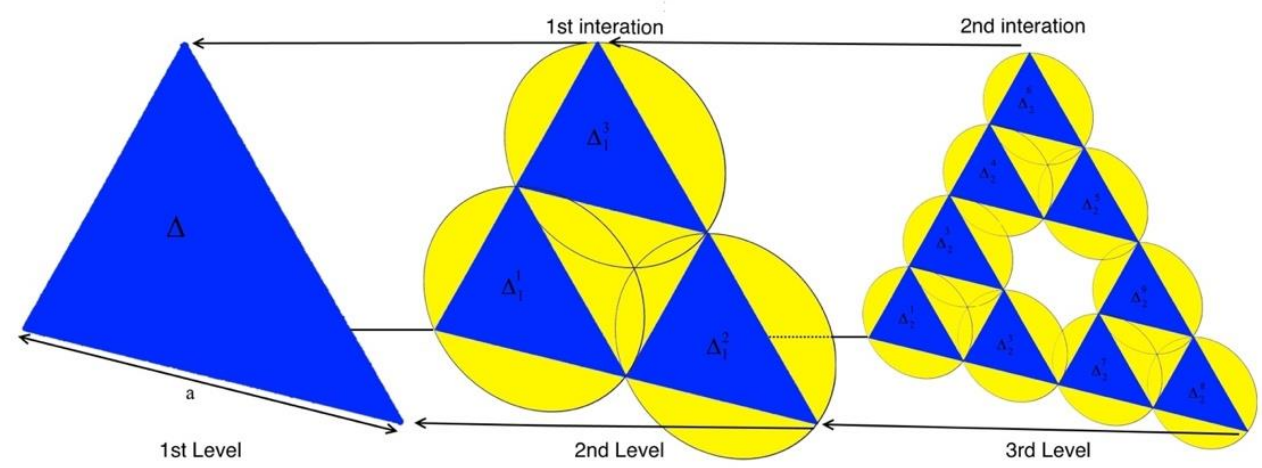

Figure 1. Geometrical representation of synergetic effect on the example of fractal conceptual model

The sum of differences of the areas on each level between the area of a circle (the minimum area containing a triangle) and the area of a triangle (the structural part of the Sierpinski triangle, which corresponds to one conceptual unit) represents assessment of the size of synergetic effect:

$$
E_{s}=\sum_{j=1,3}\left(S_{o \kappa p_{1}^{j}}-S_{\Delta_{1}^{j}}\right)+\sum_{j=1,9}\left(S_{o \kappa p_{2}^{j}}-S_{\Delta_{2}^{j}}\right)+\ldots+\sum_{j=1,3^{n}}\left(S_{o \kappa p_{n}^{j}}-S_{\Delta_{n}^{j}}\right)
$$

where the area of the circle is calculated through the side of the inscribed equilateral triangle with the side a, taken for one conventional unit. For example, at the first iteration the side of one of the three triangles is equal $1 / 2 \cdot a$, at the second $-1 / 4 \cdot a$ etc.

High-quality enrichment of ideational operations through formation of knowledge hierarchy, streamlining of contents and structure of cognitive experience, strengthening of communication and social interaction of subjects on the basis of dialogue of cultures, development of research activity, efficient self-control of personal lines of the student, strengthening of motivation of trainees will serve as a form of expression of a synergetic effect in the conditions of integration of mathematical, informational, scientific and humanitarian knowledge. 


\section{Results of the research}

Computer monitoring of students' knowledge based on the fractal methods involves the ATS following components:

- cross-disciplinary fractal and organized base of key mathematical concepts;

- the informational model for tutoring process automation is presented in the form of bank of educational and cognitive and research tasks which is coordinated with fractal structure of a conceptual framework;

- the program module focused on individual estimation of quality of educational students cognitive activity in two parameters: depth of knowledge based on The Hurst exponent and the size of synergetic effect of educational cognitive activity.

So, on the basis of the educational thesaurus the informational model for tutoring process automation presented in the form of a matrix of educational and cognitive problems in mathematics is formed. Promotion of the matrix allows to calculate probability of stay at the particular level of development of contents taking into account depth of fractal representation of educational elements and to automatically make correction of the direction of granting to consideration of the proposed material horizontally and vertically. Horizontal levels establish realization of dialogue of mathematical, humanitarian and naturally scientific cultures in the course of tutoring according to the first iteration of fractal structure of an educational element (concept): mathematical profile, naturally scientific profile, humanitarian profile. Vertically we allocate levels of digestion of training mathematical material according to further body height of fractal sets: founding of mathematical knowledge; basic fundamental mathematical knowledge; level of allvocational training (ability to apply mathematical knowledge in annexes to the sphere of professional disciplines); level of practical self-realization (personal and professional selfconsciousness, self-rating and self-development).

The model allows to correlate complexity and volume of educational information with intellectual opportunities of the trainee, level of his competence and to choose the corresponding tasks. Information on results and parameters of realization of tasks is automatically brought in accumulative bank of information on users. Realization of the majority of tasks of all levels on a vertical and a horizontal allows to receive the maximal degree of fullness of volume and depth of specification of an educational element.

Within informational model of tutoring and the allocated levels the program module of assessment and diagnosing of success of students is formed. For activization of the qualimetrical device it is necessary to define intensity and dispersion of the probability processes bringing system from one state into another which is characterized by a number of parameters, in particular rate of accumulation of educational information. Application of 
the theory of fractals allowed to solve this problem. On the basis of the offered algorithm the program module in the $\mathrm{C \#}$ programming language was developed for determination of coefficient of depth of assimilation of a concept and the mechanism of assessment of size of the synergetic effect of integration of knowledge and activity (fig. 2), focused on diagnostics of quality of knowledge of two parameters - individual estimation of depth of knowledge (D) and assessment of size of synergetic effect (Es).

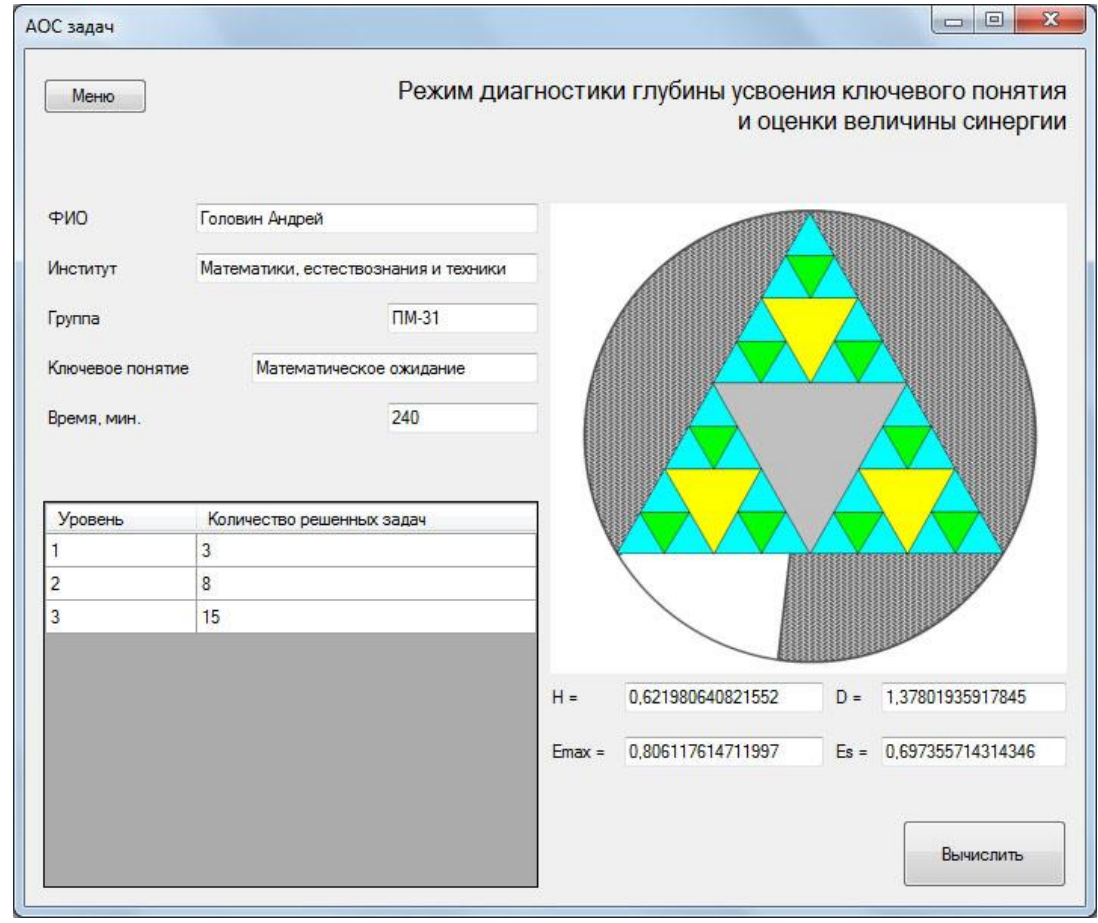

Figure 2. Module of monitoring of mathematical knowledge

The choice of a key concept, the common time allowed for studying of a concept, quantity of the acquired concepts during sampling $(\mathrm{k})$ are loaded automatically from the ATS Artificial intelligence block. Due to the fact that Ai block projected and calculated not only the quality of the learning material (completeness and extent of its assimilation, completeness of assimilation of communications of concepts with other concepts, etc.), but also the number of iterations on fractal representation of mathematical concepts which were used by the student. The visual representation of this module applies the ratio transformation of the calculated values to strengthening of obviousness and simplification of perception of information by the nonspecialist in informational technologies: 


$$
k=\frac{(4 \cdot \pi-3 \cdot \sqrt{3}) / 12}{E_{\max }} .
$$

For three levels of digestion of training mathematical material this coefficient is approximately equal $k \approx 0,7619$. For further internal calculations not scalable data are used.

\section{Discussion and conclusion}

The considerable didactic value of the developed diagnostic technique consists in the fact that application of fractal methods in projection of technology of knowledge monitoring gives the chance to increase the accuracy and speed of level evaluation of students proficiency with the possibility of identification of synergetic effect. Fractal methods for diagnostics of results of tutoring promote assimilation and fixing of cross-disciplinary knowledge, activization of various ways of information perception, excluding the possibility of mechanical learning of conceptual material, unilaterality of development and impoverishment of the emotional sphere of future experts. In development of computer monitoring of knowledge the fact that evaluation test of students tutoring (carried out on the basis of two parameters - coefficient of depth of assimilation of a concept and its interrelation) with other concepts and assessment of synergetic effect of knowledge integration and activity of trainees is essential.

Thus, the technology of assessment activity on the basis of fractal model operation of mathematical structures allows increasing effectiveness, reliability and a validity of system of a qualimetria of educational process.

\section{References}

Avanesov, V. S. (2005) Form of test tasks. M.: Testing centre.

Bolotov V. A (2005). Assessment of knowledge: main approaches to creation of the allRussian evaluation test system of education. Economy and education today. No. 3.

Dvoryatkina, S. N., Smirnov, E. I. (2016) Assessment of the synergetic effects of integration of knowledge and activity on the basis of computer model operation. The modern informational technologies and IT education. M.: MSU. 35-42.

Kibzun, A. I., Karolinskaya, S. N., Shayukov, R. I. (2006) System of distance learning on mathematical disciplines in higher education institution. Messenger of computer and informational technologies, 4, 99-106.

Latyshev, V. L. (2009) Criteria for evaluation of quality of an educational component of the intellectual training systems. Informatization of science and education, 3, 89-96.

Myakishev, V. V., Semenko, V. I. (2002) Monitoring of knowledge in system of distance learning. Problems of management of economic systems, 10. 
Rudinskiy, I. D (2005) Training of specialists in the field of formal and structural description, research and organization of pedagogical testing of knowledge: dis. ... Dr. ped. Sciences: 13.00.02. - AM, p. 448.

Robert, I. V., T. A. Lavin T. A. (2012) Explanatory dictionary of terms of a conceptual framework of informatization of education; M.:The BKL Publishers.

Robert I.V. (2010) Modern information technologies in education: didactic problems; Perspectives of use. M .: IIO RAO.

Uglev (2010) About the specifics of individualization of tutoring in the automated training systems. Education philosophy, 2, 68-74.

Shmelyov, A. G. (2013) Practical testology: testing in education, applied psychology and human resource management. M.: Mask. 